\title{
Displacement cross sections of electron irradiated graphene and carbon nanotubes
}

\author{
Qiang Yan ${ }^{\mathrm{a},}$, Jing Wang ${ }^{\mathrm{c}}$, Di Chen ${ }^{\mathrm{b}}$, Jonathan Gigax ${ }^{\mathrm{b}}$, Lin Shao ${ }^{\mathrm{b}, \mathrm{c}, *}$ \\ ${ }^{a}$ College of Nuclear Science and Technology, Harbin Engineering University, Harbin, 150001, P.R. \\ China \\ ${ }^{\mathrm{b}}$ Department of Nuclear Engineering, Texas A\&M University, College Station, TX 77843, USA \\ ${ }^{c}$ Department of Materials Science and Engineering, Texas A\&M University, College Station, TX 77843, \\ USA
}

\begin{abstract}
We calculate the displacement cross-sections (DCS) of low dimensional carbon systems under electron irradiation and present an analytical formula being able to evaluate displacement creations under various conditions. The calculations consider Mott scattering, charge screening effects and thermal vibrations of target atoms. DCS values of graphene and single-walled carbon nanotubes (SWNTs) as a function of electron beam energies, substrate temperatures, and tube diameters are calculated through combination with carbon threshold displacement energies obtained from molecular dynamics simulations. For SWNTs, the smaller the tube diameters the higher the DCS values. Such diameter dependence is the most pronounced for low energy electron beams. Furthermore, DCS values are most sensitive to temperatures when electron beam energy is low. However, the temperature sensitivity disappears at higher electron energy, specifically at $200 \mathrm{keV}$ and beyond.
\end{abstract}

Keywords: Displacements; electron irradiation; graphene; carbon nanotubes

*Corresponding author at: Department of Nuclear Engineering, Texas A\&M University, College Station, TX 77843-3133, United States. Tel.: +1 979845 4107; fax: +1 9798456443.

E-mail address: 1shao@tamu.edu (L. Shao). 


\section{Introduction}

Defect engineering of low dimensional carbon systems such as graphene and carbon nanotubes (CNTs) are important for various device applications [1-4]. Unique doping and structural modification have been reported under electron and ion bombardments [5-8]. Understanding of displacement creation and damage cascade evolution under particle irradiation are important for further tuning material properties. However, damage calculations in carbon materials have been largely influenced by uncertainty and scattered data from both modeling and experimental studies, as well as the complexity caused by geometry differences among different carbon nanostructures. For example, the threshold displacement energy $\left(E_{d}\right)$ default value is $28 \mathrm{eV}$ in the Monte Carlo simulation code SRIM for carbon materials [9], but experimental studies suggest $E_{d}$ should be about 15-20 eV for CNTs [10] and 18-20 eV for graphene [11]. For modeling studies on graphene, Banhart et al. reported $22 \mathrm{eV}$ using a dynamic model and $15 \mathrm{eV}$ using a static model [12]. One suggested average is $22.2 \pm 0.2 \mathrm{eV}$ [13]. Molecular dynamics (MD) simulations on CNTs of different tube diameters suggest that $E_{d}$ decreases from $22 \mathrm{eV}$ for large diameter CNTs to 15 $\mathrm{eV}$ for the smallest CNT tubes [10,14]. On the other hand, the observation of CNT structural changes under bombardments of a $200 \mathrm{keV}$ electron analysis beam in a transmission electron microscopy (TEM) chamber suggest that the true displacement energy could be even lower [14]. Recent modeling shows that carbon displacement is facilitated when electron momentum transfer direction is aligned with carbon thermal vibration direction, thus the threshold electron beam energy for displacement creation is further reduced $[8,15]$.

Both fundamental studies and technological developments require high accuracy in calculating displacement creation in graphene and CNTs under electron irradiations, particularly in the energy region of $100 \mathrm{keV}$ to $1 \mathrm{MeV}$ since they are typical beam energies used by TEM. Irradiation effects need to be minimized as unwanted disturbance to original structures in many TEM characterization experiments. But in other experiments beam-induced effects are intentionally introduced for in situ electron irradiation and TEM characterization, in order to study radiation responses and subsequent dynamic structural evolutions 
of irradiated TEM specimens [5]. In both cases, calculations of carbon displacement numbers are needed. The calculations, however, are complicated and need to consider electron screening effects, relativistic energy transfer and thermal vibration of target atoms. Ideally, an analytic formula should be developed with flexibility for describing various electron irradiation conditions.

\section{$2 \quad$ Modeling procedure}

The present study aims to calculate displacement cross-sections (DCS) by considering the complexity mentioned above. First, we calculate the screened Mott differential cross-sections, which give differential cross-sections under specific scattering configurations. Next, the energy transfer from electrons to carbon target atoms, including the influences from thermal vibration, are calculated under specific energy and scattering angle. Then, the total displacement cross-section is calculated through integration of the crosssections corresponding to energy transfer larger than threshold displacement energies $E_{d}$. The integration additionally considers thermal vibration probability distributions from Debye model.

\subsection{Screened Mott differential cross section}

The first theoretical description of differential scattering cross-sections between a nucleus and a relativistic electron was given by Mott [16]. Unfortunately, the final expression is an infinite series Legendre expansion. Towards more realistic usage, many numerical approximations have been proposed [17-21]. One common approach is to modify Rutherford cross-sections obtained from binary collisions. Furthermore, the screening effect of atomic electrons must be considered [22-27]. The general expression of differential scattering cross section, is given by

$$
\frac{d \sigma_{m o t t}}{d \Omega}=R_{m o t t} \times \frac{d \sigma_{R u t h}}{d \Omega} \times\left[1-F_{e}(q)\right]^{2}
$$


where the term $\left[1-F_{e}(q)\right]^{2}$ is the screening factor, $R_{\text {mott }}$ is the ratio between Mott differential cross sections and Rutherford differential cross sections . The latter is expressed by R.Idoeta and F. Legarda as [20]:

$$
\frac{d \sigma_{\text {Ruth }}}{d \Omega}=\left(Z \times r_{e}\right)^{2} \times\left(\frac{1-\beta^{2}}{\beta^{4}}\right) \times \frac{1}{(1-\cos \theta)^{2}}
$$

where $\theta$ is the scattering angle of electrons, $\beta$ is normalized electron velocity $v / c$ ( $v$ is the velocity of electrons, and $\mathrm{c}$ is the speed of light in vacuum), $\mathrm{Z}$ is the atomic number of target atom, and $\mathrm{r}_{\mathrm{e}}$ is the classic radius of an electron $\left(\mathrm{r}_{\mathrm{e}}=2.817938 \times 10^{-13} \mathrm{~cm}\right)$. The expression for $R_{\text {mott }}$ is much more complicated. One widely used analytical expression was given by Mckinley and Feshbach, but it is valid only for low Z materials ( $\mathrm{Z} \leq 29)$. For high $\mathrm{Z}$ materials, tabulated data is needed to calculate $R_{\text {mott }}[21,28,29]$. Boschinia et al. proposed the following format to cover a wider electron energy range (1 keV to $900 \mathrm{MeV})$ and more general target elements $(1 \leq \mathrm{Z} \leq 118)$ with 30 coefficients provided for each element [29],

$$
\begin{aligned}
& R_{\text {Mott }}=\sum_{j=0}^{4} \alpha_{j}(Z, \beta)(1-\cos \theta)^{\frac{j}{2}} \\
& \alpha_{j}(Z, \beta)=\sum_{k=1}^{6} b_{k, j}(Z)(\beta-\bar{\beta})^{k-1}
\end{aligned}
$$

Under Dirac-Hartree-Fock-Slater screening model, the $F_{e}(q)$ term in Eq. 1 can be expressed as [27]:

$F_{e}(q)=\sum_{i=1}^{3} A_{i} \frac{\left[h a_{i} /(2 \pi)\right]^{2}}{\left[h a_{i} /(2 \pi)\right]^{2}+q^{2}}$

where $h$ is the Plank constant, and $q$ is the momentum transfer depending on scattering angle and electron kinetic energy. Detail expression of $q$ and tabulated parameters of $A_{i}$ and $\alpha_{i}$ are provided in [27].

\subsection{Energy transfer from electrons to target atoms and displacement cross section}


For collisions with a static target atom without considering thermal vibration, the energy transfer from relativistic electron, $E_{t}$, is calculated by

$E_{t}=\frac{\left[\left(E_{k}+m_{e} c^{2}\right) \sin ^{2} \theta+M c^{2}(1-\cos \theta)\right] E_{k}\left(E_{k}+2 m_{e} c^{2}\right)}{\left(E_{k}+M c^{2}\right)^{2}-E_{k}\left(E_{k}+2 m_{e} c^{2}\right) \cos ^{2} \theta}$

where $\theta$ is electron scattering direction, $E_{k}$ is the kinetic energy of electrons, $M$ is the mass of target atom, and $m_{e}$ is electron mass. If thermal vibration is considered, the final expression of $E_{t}$ for relativistic electrons is very complicated. In the framework of special relativity, the 4-vector momentum is defined as $P^{u}=\left[E, P_{x} c, P_{y} c, P_{z} c\right]$, where E, P, and c, are energy, momentum, and speed of light, respectively. Based on the momentum conservation, $\vec{P}_{a}+\vec{P}_{b}=\vec{P}_{c}+\vec{P}_{d}$, where $\vec{P}_{a}, \vec{P}_{b}, \vec{P}_{c}$, and $\vec{P}_{d}$ are momentum of the electron and the target atom before and after scattering, we obtain the following equation,

$$
\begin{aligned}
\left(m c^{2}+M c^{2}+\right. & \left.E_{k}+E_{\text {thermal }}\right) E_{t}+m^{2} c^{4}-\left(m c^{2}+E_{k}\right)^{2} \\
& -\sqrt{E_{k}\left(E_{k}+2 m c^{2}\right) E_{\text {thermal }}\left(E_{\text {thermal }}+2 M c^{2}\right)} \cos \alpha \\
& +\left[\sqrt{E_{k}\left(E_{k}+2 m c^{2}\right)} \cos \theta+\sqrt{E_{\text {thermal }}\left(E_{\text {thermal }}+2 M c^{2}\right)}(\cos \alpha \times \cos \theta+\sin \alpha\right. \\
& \times \sin \theta)] \sqrt{\left(E_{k}-E_{t}\right)\left(E_{k}-E_{t}+2 m c^{2}\right)}=0
\end{aligned}
$$

where $\alpha$ is target atom's original trajectory before collision, $E_{\text {thermal }}=0.5 M v^{2}$ is thermal vibrational energy of a target atom and $\mathrm{v}$ is vibrational speed of atoms. The Eq. (6) is based on the condition that the target atom's thermal vibration direction is aligned with the electron momentum direction, and the likelihood is determined by the probability distribution under Debye mode. It is worth noting that Meyer et al. provided a simplified analytic expression for $E_{t}$, the detailed of derivation of which was not given [15]. The solutions in the present study are obtained by numerically solving Eq. 6, and the results are compared to Meyer et al. [15]. The total displacement cross-section in both methods is calculated by 
$\sigma=\int_{-\infty}^{\infty} p(v) \sigma_{d}(v) \Theta(v) d v$

where $v$ is vibrational speed of atoms, $p(v)$ is the probability distribution of speeds and dependent on the Debye temperature of the target atom [15], and $\sigma_{d}(v)$ is total cross-sections to scatter target atoms with energies larger than $E_{d}$. Atomistic vibrations play a role in the recoiled energy $E_{t}$, as reflected by the dependence of $E_{t}$ on $E_{\text {thermal }}$ in Eq. 6. $\Theta(\mathrm{v})$ is a function to ensure $E_{t}>E_{d}$, and equals 1 when $E_{t}>E_{d}$, and 0 when $E_{t}<E_{d}$. Eq. 7 is based on the Debye model and is valid in the temperature region of $\mathrm{T} \geq 150 \mathrm{~K}$. At $\mathrm{T}<150 \mathrm{~K}$, the resonance and isomer shifting invalidate both the Debye model and Einstein model $[15,29]$.

\section{$3 \quad$ Results and discussions}

Fig. 1 shows the comparison of differential cross-sections of electron-carbon scattering, obtained from the present study by using Eq. (1), and from the code ELSEPA [30], and NIST database [31]. Both ELSEPA and NIST data use the partial wave approximation. Good agreement among the three sets of data suggests that our calculation based on tabulated data is accurate when compared with widely accepted data. It is worth noting that ELSEPA and NIST cross sections are valid for $\mathrm{Z} \leq 92$, while tabulated data are valid for $\mathrm{Z} \leq 118$ and $\mathrm{E} \leq 900 \mathrm{MeV}$.

Fig. 2 compares the maximum kinetic energy transferred as a function of scattering angles for carbon target atoms scattered by $40 \mathrm{keV}$ electrons at temperatures of $300 \mathrm{~K}$ and $3500 \mathrm{~K}$ (close to the melting point of graphite), respectively. Comparisons are made for three cases: static atoms without thermal vibration (from Eq. 5), vibrating atoms considering the temperature effect (from Eq. 6), and an earlier model considering thermal vibrations [15]. Different from static atoms which have a single-valued transferred energy after scattering, thermally vibrating atoms gain kinetic energy in a range due allowable vibration velocities and directions. The maximum kinetic energy transfer considering thermal vibrations is higher than that without considering vibrations, which is the main cause that thermal vibration is able to reduce 


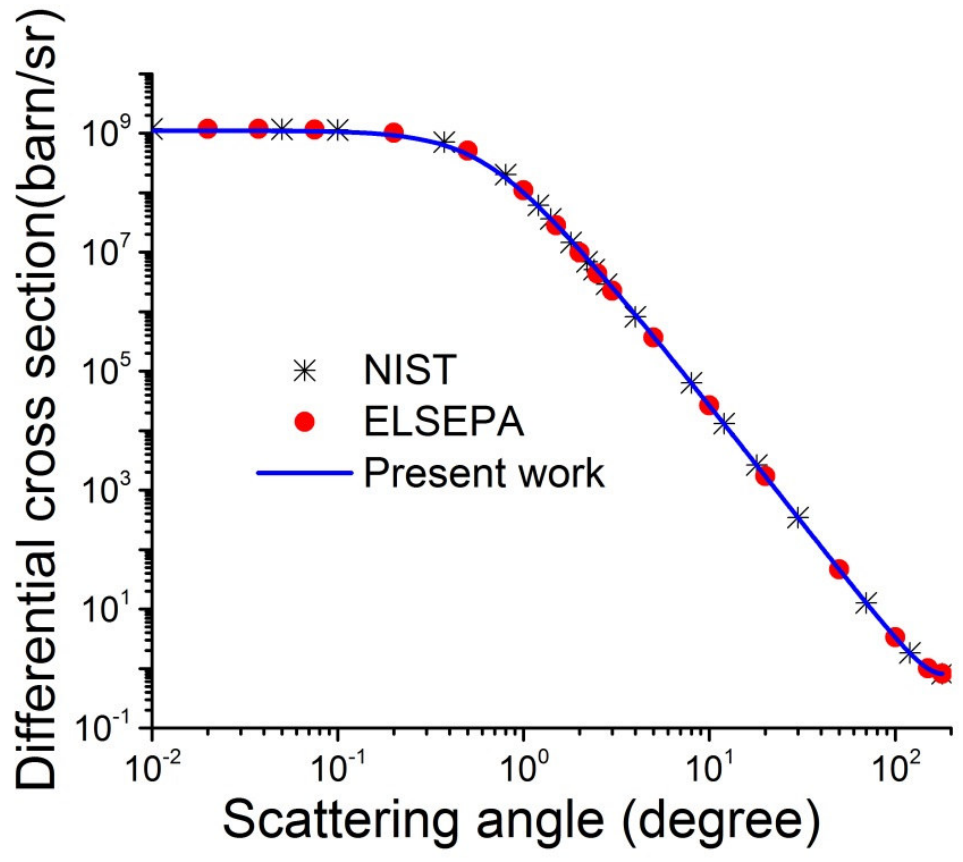

Figure 1. Differential scattering cross-sections of $200 \mathrm{keV}$ electrons in carbon, obtained from the NIST database, ELSEPA code and the present work.

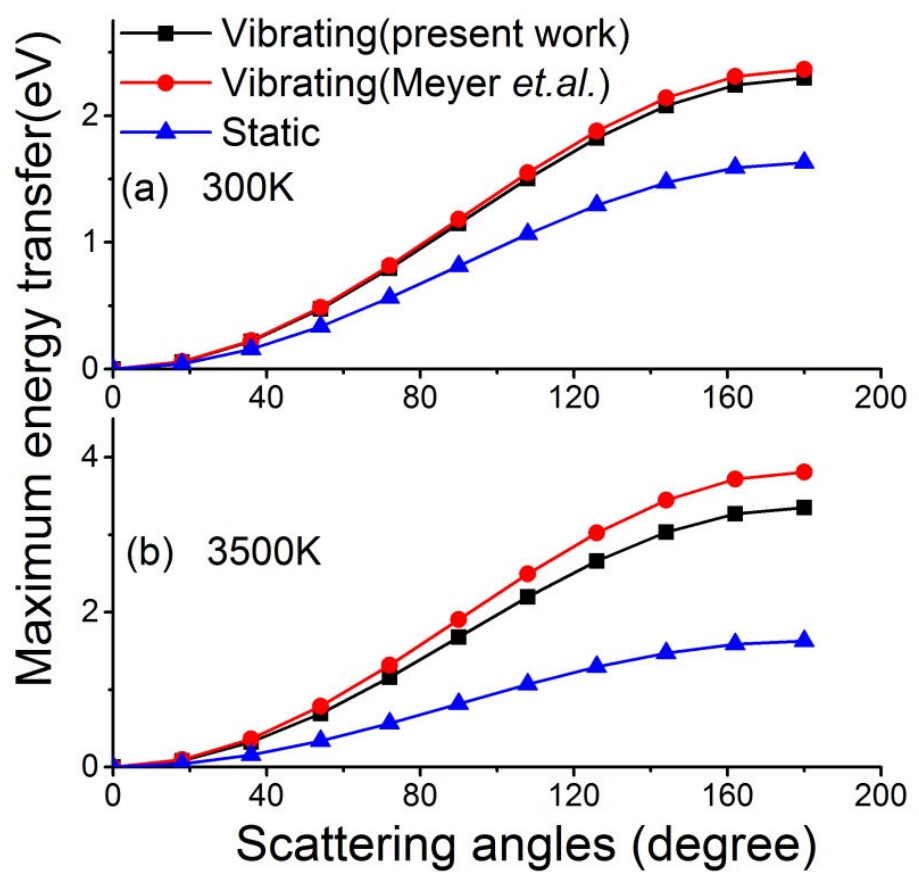

Figure 2. The maximum kinetic energy transferred from $40 \mathrm{keV}$ electrons to carbon atoms, calculated with or without considering thermal vibration. 
the minimum electron energies required for carbon displacement creation, as pointed by Meyer et al. The maximum energy transfer occurs when one incident electron has a head-on collision with one carbon atom whose thermal vibration direction is aligned with the electron's incident direction. Our curve obtained, based on tabulated data, is slightly higher than that from Meyer et al.[15]. We attribute the difference to the fact that the formula provided in the previous study uses certain approximations in deriving an analytic expression. The difference is relatively large at higher temperatures, as shown in Fig. 2(b), and is negligible at low temperatures (i.e. 300K). Overall, both curves are in reasonable agreement.

In order to provide an overview picture on displacement creation efficiency under different electron bombardment conditions, we calculate DCS values as a function of $E_{d}$ and electron energies, $E_{k}$. All data are obtained by using tabulated parameters. In Fig. 3(a), the influences of $E_{d}$ on DCS values at a fixed temperature of $300 \mathrm{~K}$ are compared. The lower the $E_{d}$, the higher the saturated DCS values at large $E_{k}$. Saturation is reached around $E_{k} \sim 250 \mathrm{keV}$, and the transition to the saturated regions is weakly dependent on electron beam energies. On the other hand, DCS values quickly increase between energies of $\sim 70 \mathrm{keV}$ to $\sim 100 \mathrm{keV}$. At much lower electron energies, the DCS values are nearly reduced to zero, which implies that in order to create enough displacements to produce a defect structure or amorphization, the required electron fluences in the low energy region cannot be reasonably achieved in typical TEM imaging analysis. Therefore, the energy at which DCS start to rise can be described as threshold electron energy for creating noticeable radiation damage. Using this criteria and $E_{d}=22 \mathrm{eV}$, the minimum displacement energy for graphene, we obtain a threshold value of $95 \mathrm{keV}$, which is very close to experimental observations [32].

To maximize the impact on realistic applications and eliminate the need for tabulated data, we fit the two dimensional mapping Fig. 3a, and obtain the following formula to predict DCS at $300 \mathrm{~K}$ : 
$D C S($ barn $)=d+A \times \exp \left(\frac{E_{k}(k e V)-4.3043 \times E_{d}(e V)+7.4348}{t}\right)$

$d=-7.36+\frac{532.32}{E_{d}(e V)}$

$A=12.71-\frac{694.31}{E_{d}(e V)}$,

$t=-175.24+\frac{2034.6}{E_{d}(e V)}$

where $\mathrm{d}, \mathrm{A}, \mathrm{t}$ are the fitting obtained parameters. The fitting is valid for $14 \mathrm{eV} \leq E_{d} \leq 28 \mathrm{eV}$. The DCS values decrease with decreasing $E_{k}$, and reduce down to zero when $E_{k}$ is less than a critical energy value. Data obtained by using Eq. (8) are plotted as solid lines in Fig. 3a for comparisons. Fitting agrees well with exact numerical values calculated from Eq. (7). Our interest is to find a universial fitting which can be applied to a $E_{d}$ energy region as wide as possible. Inaccuracy is introduced for relatively samall $E_{d}$ energies, leading to the maximum deviation about $15 \%$ when DSC values start to approach a saturated value. In other words, the fitting curve is less sharper when compared with the exact values near the start of the saturation region. The fitting, however, does not bear any physical significance.

Overall, Eq. (8) provides an efficient and reliable approach to quickly estimate DSC values, and avoids complicated numcrical calculations using Eq(7). Furthermore, as shown in Fig. 3b, our fitting suggests that the threshold electron energy (or minium energy required for displacement creation), $\mathrm{E}_{\mathrm{th}}$, can be fitted well by a linear relation given by

$E_{t h}(k e V)=4.0126 E_{d}(e V)+7.1625$ 


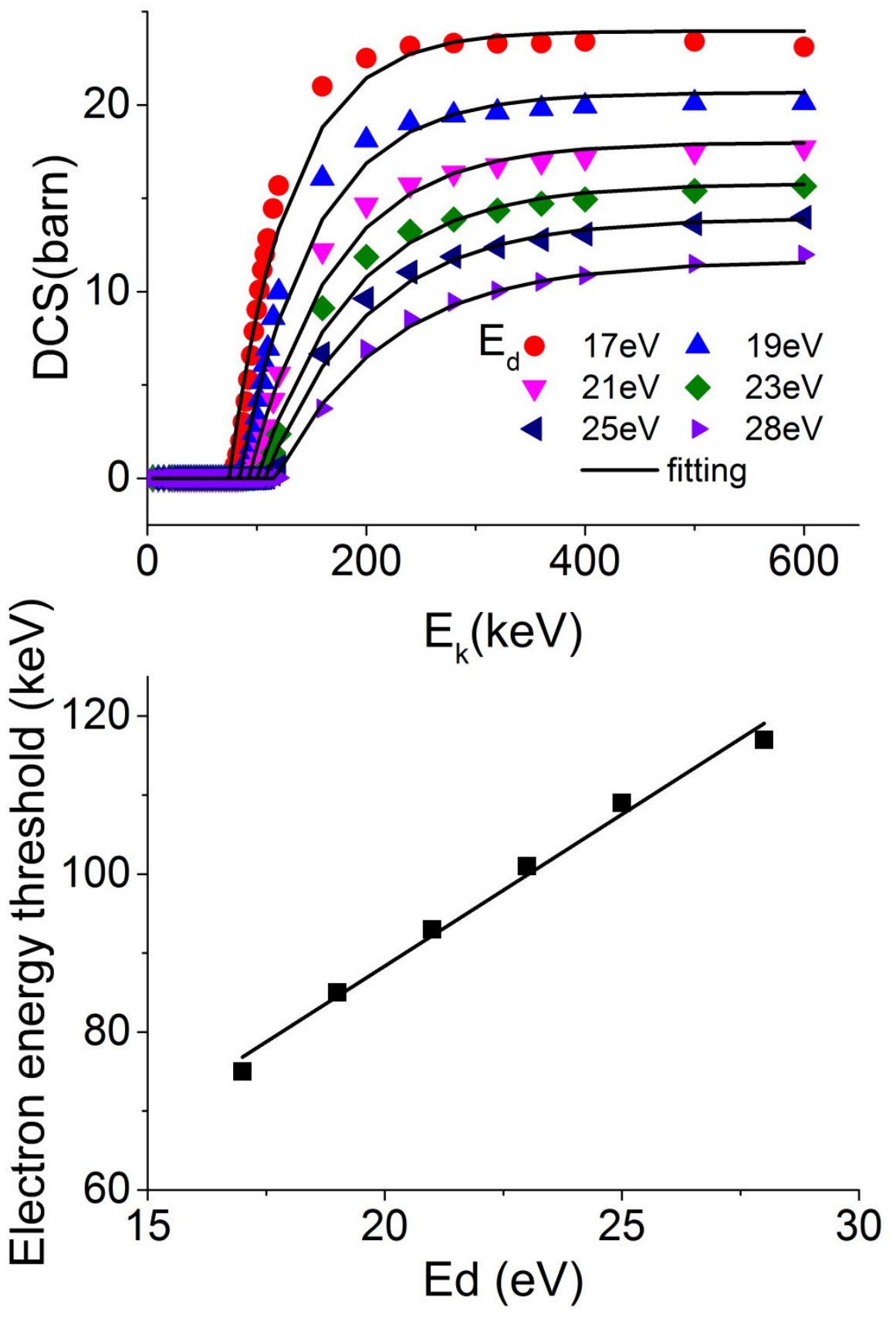

Figure 3(a). DCS as a function of selected $E_{k}$ and $E_{d}$ values, and $(b)$ threshold $E_{t h}$ as a function of $E_{d}$. The solid lines in (a) are obtained using Eq. (8) and the solid lines in (b) are obtained using Eq. (9). 
In Fig. 4a, the influence of temperature on DCS values, at a fixed displacement energy of $22 \mathrm{eV}$, are compared. Overall the temperature effects are small, in particular for electron energies more than $\sim 120 \mathrm{keV}$. For low electron energy regions $\left(E_{k} \leq 100 \mathrm{keV}\right)$, as the temperature is increased, the DCS values increase while the threshold energy decreases. In Fig. 4b, we further compare the DCS curves obtained from the present work (considering thermal vibration) and previous studies with or without thermal vibration considerations. It is clear the static model without considering thermal vibration significantly overestimates the $E_{t h}$ (at about $110 \mathrm{keV}$ ). For the current study and previous work by Meyer et al., there are no significant differences and the present work is slightly lower by $10 \%$ to $20 \%$ in the region of 90 $\mathrm{keV}$ to $110 \mathrm{keV}$. However, both vibration models suggest that an electron energy $\mathrm{E}_{\mathrm{k}}>95 \mathrm{keV}$ is needed to create significant damage.

We extend the studies to single walled carbon nanotubes. Different from carbon atoms on a graphene, the displacement energy of SWNT atom depends on the tube diameter. Previous studies by Krasheninnikov et al [14] have shown that the displacement energies change from $22 \mathrm{eV}$ for large diameter of about $17 \mathrm{~nm}$, to $15 \mathrm{eV}$ for small diameter of about $4 \mathrm{~nm}$. The reduced displacement energies are due to curvature effects. By using LAMMPS code [33], we calculate the displacement energy dependence on tube diameter, which is presented in Fig. 5. The calculation uses CBOP (Long-range Bond Order Potential) carbon inter-atomic potentials and considers the influence from neighboring carbon atoms [34]. In our studies, the structural relaxation is included when a displaced carbon atom moves across the displacement energy saddle points. Fig. 5 plots the obtained displacement energy as a function of tube diameters from $4 \mathrm{~nm}$ to $35 \mathrm{~nm}$.

Another important parameter is Debye temperature. It is generally accepted that CNT has an axial Debye temperature of $960 \mathrm{~K}$ and a transverse Debye temperature of $14 \mathrm{~K}$ [35]. Since most ion and electron irradiations are performed with the beam perpendicular to the tube axis, we use the transverse Debye temperature for the calculation. 


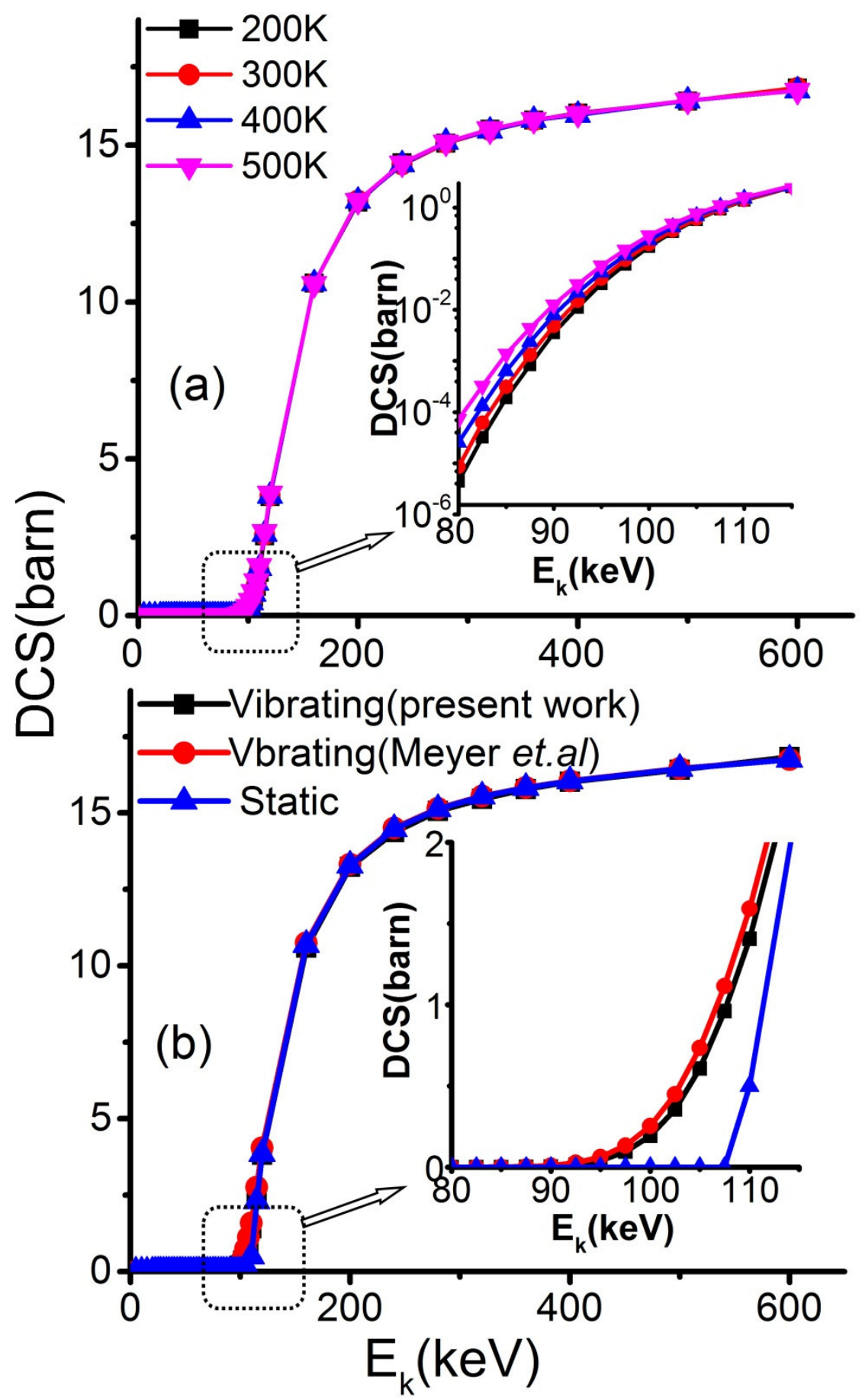

Figure 4 (a) DCS as a function of electron beam energy $E_{k}$, calculated by using displacement energy of $22 \mathrm{eV}$ and at different temperatures when thermal vibration is considered, and (b) comparisons of DCS calculated with or without thermal vibration at $300 \mathrm{~K}$. 


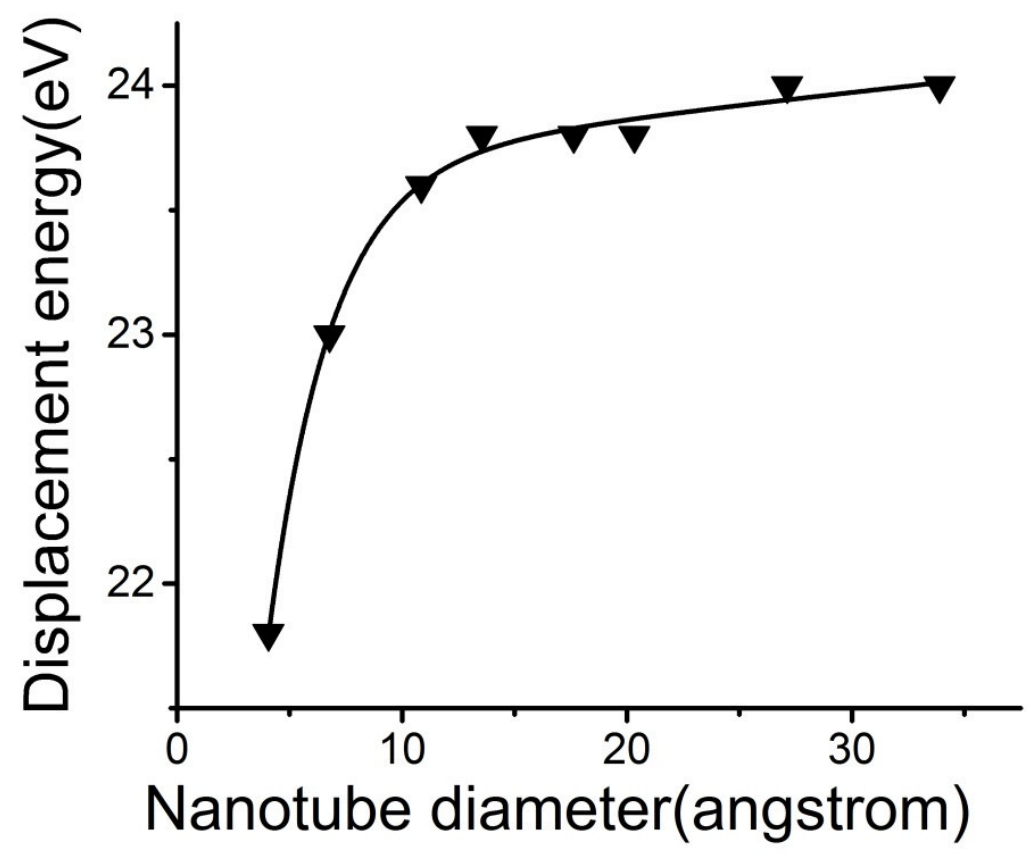

Figure 5. Displacement energies as a function of tube diameters in SWNTs, calculated by MD simulations.

Our obtained DCS data as a function of temperature and tube diameters are plotted in Fig. 6a-d for electron beam energies of $80 \mathrm{keV}, 100 \mathrm{keV}, 120 \mathrm{keV}$ and $200 \mathrm{keV}$, respectively. The tube diameter dependence is most significant for low electron energies. As shown in Fig. 6a, the DCS values of smallest tube is about four order of magnitudes higher than largest tube under irradiation by $80 \mathrm{keV}$ electron beam at room temperature. With increasing electron energies, the difference reduces to about two order of magnitudes under $100 \mathrm{keV}$ electron beam irradiation (as shown in Fig. $6 \mathrm{~b}$ and about a factor of 4 for 120 $\mathrm{keV}$ (as shown in Fig. 6c) and to approximately 30\% for $200 \mathrm{keV}$ (as shown in Fig. 6d). Furthermore, the temperature effects are also sensitive to electron beam energies. The lower the electron beam energies, the larger the DCS differences. From $200 \mathrm{~K}$ to $500 \mathrm{~K}$ for the lowest electron energy $(80 \mathrm{keV})$, the DCS values of large tubes increase by about two orders of magnitudes. For highest electron energy (200 keV), all curves converged. 


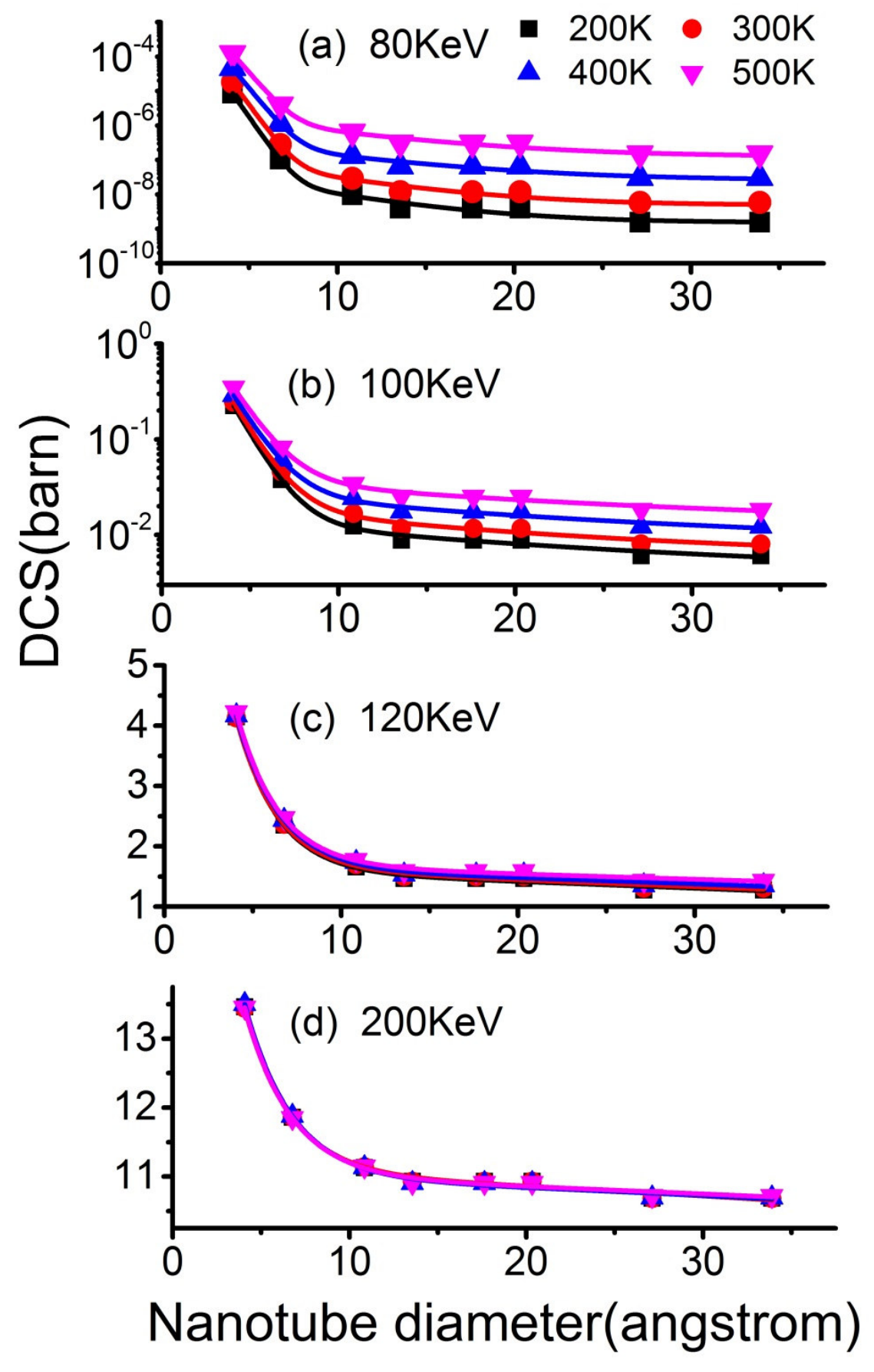

Figure 6. DCS values as a function of SWNT tube diameters for temperatures of 200, 300, 400, and $500 \mathrm{~K}$, and for electron beam energies of (a) $80 \mathrm{keV}$, (b) $100 \mathrm{keV}$, (c) $120 \mathrm{keV}$, and (d) $200 \mathrm{keV}$, respectively. 
Fig. 6 further shows the following features: Regardless of tube diameters and temperatures, the DSC values are less than 10-3, 1, 5, and 15 barns, for electron beam energies of 80, 100, 120, and $200 \mathrm{keV}$, respectively. For a typical TEM electron beam energy of $200 \mathrm{keV}$, the DSC values are larger than 10 barns, regardless of the tube diameters. For large diameters and at room temperature, the DSC values increase by about 9 orders of magnitudes if electron beam energies increase from $80 \mathrm{keV}$ to $200 \mathrm{keV}$. For large diameters, DSC values of SWNT approach that of a graphene sheet.

The conclusion that the threshold electron energy is about $80 \mathrm{keV}$ agrees with earlier experimental observations. Smith and Luzzi observed no damage at electron beam energy less than $80 \mathrm{keV}$ [36]. These authors also used a static model to obtain a $E_{t h}$ of $86 \mathrm{keV}$. Their calculation used displacement energies obtained from tight binding MD simulations [37], which is systematically lower than values obtained by us performing structural relaxation during carbon atom recoiling. Furthermore, screening effects in binary knock-on scattering were not considered [38], which lead to an overestimation on the maximum energy transfer and, consequently, a much lower threshold electron energy even without considering thermal vibration. Yasuda et al. considered the screening effect but not thermal vibration effect, and obtained slightly overestimated electron threshold energies; about $110 \mathrm{keV}$ for $(10,10)$ SWNTs. The definition of indices $(n, m)$ of SWNT can be found in Ref. 39. These authors incorporated the DCS data into a Monte Carlo code and further linked the code to MD simulations [40].

The current studies are limited to SWNTs but a strong displacement energy dependence in multiwalled nanotubes (MWNTs) is expected. In addition to influence of neighboring carbon atoms on the same tube plane, carbon displacement is affected by carbon atoms in the neighboring tube planes. In other words, displacement energy is expected to be different for the middle, innermost and outermost tubes, even under the same tube diameter. For a large MWNT, the difference in DCS values from the innermost tube to the outermost tube is expected to lead to preferred sputtering or collapse of the innermost tubes. 
Our studies further show that such behavior from radiation damaging process is favored for low energy electron beam due to the orders of magnitude differences in DCS values.

Determination of DCS values by considering factors such as electron energies, temperature and tube diameters is important for characterization techniques such as TEM when appropriate electron beam conditions must be selected to minimize damage and disturbance to carbon systems. Furthermore, considerations must be made for beam modification techniques when displacements, amorphization, tube shrinkage and tube joining are desired.

\section{Conclusion}

Displacement cross-sections of electron irradiated graphene and SWNT are calculated by considering screening effect and thermal vibration. First, screened Mott differential cross sections are obtained under specific electron-carbon scattering angles. Next, the energy transfer from incident electron to target carbon atom is calculated under the same scattering geometry. Then, the total DCS is calculated by integration of differential cross-sections with transferred energy larger than the displacement energies. Through fitting, an analytical formula is provided to calculate displacement cross sections. For graphene and SWNT of different diameters, displacement energies are calculated by using molecular dynamics simulations, and the data is used as an input into DCS calculations. Thermal vibration reduces the threshold electron energy for displacement creation in both graphene and SWNTs, in agreement with earlier modeling results based on different cross section treatments. For SWNT of different tube sizes, a small $(5,5)$ tube is easier to be damaged and its DCS values are a few orders of magnitudes higher than that of a large $(15,15)$ tube. Such DCS differences are more significant at lower electron beam energy. For different temperatures, the curves of DCS dependence on tube diameters are symmetrically shifted upwards at high temperatures. Relative curve shifting between tube diameters is high for low energy 
electron beam and becomes negligible for high energy electron beam. This study identifies the key parameters which influence the damage creation in low dimensional carbon systems.

\section{Acknowledgements}

This work was supported by the US Department of Energy, Office of Basic Energy Sciences, under Grant No. DE-SC0006725. 


\section{Reference:}

[1] J. Lahiri, Y. Lin , P. Bozkurt, I. I. Oleynik, and M. Batzill, Nat. Nanotech. 5 (2010) 326-329.

[2] M.M. Ugeda, I. Brihuega, F. Hiebel, P. Mallet, J.Y. Veuillen, et.al.,Phys. Rev. B 85 (2010) 121402.

[3] M. Sammalkorpi, A. Krasheninnikov, A. Kuronen, K. Nordlund, and K. Kaski Phys. Rev. B 70 (2004) 245416.

[4]S. L.Mielke, D. Troya, S. L.Zhang, J.L.Li, S.P.Xiao, et al,Chem. Phys. Lett. 390 (2004) 413-420.

[5]A.V. Krasheninnikov and F. Banhart, Nat. Mater. 6 (2007) 723-733

[6] A.W. Robertson, C.S. Allen, Y. A. Wu,K.He, J. Olivier, et al. Nat. Comm. 3 (2012) 1144.

[7] A. Aitkaliyeva, D. Chen,and L. Shao, Sci. Rep. 3 (2013) 2774.

[8]J. Wang, D. Chen, J. Wallace, et al. Appl. Phys. Lett. 104 (2014) 191902.

[9]J.F. Ziegler, M.D. Ziegler and J.P. Biersack, Nucl. Instr. Meth. in Phys. Res. B 268 (2010)

[10]F. Banhart, Rep. Prog. Phys. 62 (1999) 1181-1221.

[11] F. Banhart, J. Kotakoski and A.V. Krasheninnikov, ACS Nano 5 (2011) 26- 41.

[12] F. Banhart, J. X. Li, and A. V. Krasheninnikov, Phys. Rev. B 71 (2005) 241408(R).

[13] O. Lehtinen, J. Kotakoski, A.V. Krasheninnikov, Phys. Rev. B 81(2010)

[14] A. Krasheninnikov, F. Banhart, J. Li, A. Foster, and R. Nieminen,Phys. Rev. B 72 (2005)

[15]J.C. Meyer, F.Eder, et al, Phys. Rev. Let. 108 (2012) 196102.

[16]N. F. Mott, Proc. Roy. Soc. A 124 (1929) 425-442.

[17]W.A. McKinley and H. Feshbach, Phys. Rev. 74 (1948) 1759.

[18]H. Feshbach, Phys. Rev. 88 (1952)295.

[19] N. Sherman, Phys. Rev.103 (1956) 1601.

[20] R. Idoeta and F. Legarda, Nucl. Instr. Meth. Phys. Res. B 71 (1992) 116-125

[21] L.J. Teng, Q. Hou and Z.G. Luo, Rad. Phys. Chem. Vol. 45 (1995) 235-245

[22] S. Lin and N. Sherman, Nuclear Physics 45 (1965) 492-504

[23] A.F. Bielajew, Nucl. Instr. Meth. Phys. Res. B 86 (1994) 257-269

[24]J.M. Fernandez, R. Mayol and F. Salvat, Nucl. Inst. Meth. Phys. Res. B 82 (1993) 39-45 
[25] E. Huttel , W. Arnold, H. Baumgart, Nucl. Instr. Meth. Phys. Res. B 12 (1985) 193-199

[26] E. Zeitler and H. Olsen, Phys. Rev. 136 (1964) A1546.

[27] F. Salvat, J.D. Martinez, R. Mayol and J. Parellada, Phys. Rev. A 36 (1987) 467.

[28]Z. Czyzewski, D.O. MacCallum, A.D. Romig, and D.C. Joy, J. Appl. Phys. 68 (1990) 3066-3072.

[29]M. Boschinia, J. Consolandi, M. Gervasia, et al. J. Rad. Phys. Chem. 90 (2013) 39-66.

[30] F. Silvat, A. Jablonski, C. Powell, Com. Phy. Com. 165 (2005)157-190.

[31] http://www.nist.gov./srd/nist64.cfm

[32] B.W. Smith, D.E. Luzzi, J. Appl. Phys. 90(2001)3509-3515.

[33] http://lammps.sandia.gov

[34] J. H. Los and A. Fasolino, Phys. Rev. B 68 (2003) 024107.

[35] J. Honse, in Carbon nanotubes (eds. M.S. Dresselhaus, G. Dresselhaus, and Ph. Avouris), Topics Appl. Phys. 80, 273-286 (2001).

[36]B.W. Smith and D.E. Luzz, J. Appl. Phys. 90 (2001) 3509.

[37]V.H. Crespi, N.G. Chopra, M.L. Cohen, A. Zettl, and S.G. Louie, Phys. Rev. B 54 (1996) 5927.

[38]R.S. Averback and T.D. de la Rubia, Solid State Physics, Academic, New York, 1997, Vol. 51, pp. $281-402$

[39] R. Saito, M. Fujita, G. Dresselhaus, M.S. Dresselhaus, Appl. Phys. Lett. 60 (1992) 2204.

[40]M. Yasuda, Y. Kimoto, K. Tada, H. Mori, S. Akita, et.al, Phys. Rev. B 75 (2007) 205406. 


\section{Captions:}

Figure 1. Differential scattering cross-sections of $200 \mathrm{keV}$ electrons in carbon, obtained from the NIST database, ELSEPA code and the present work.

Figure 2. The maximum kinetic energy transferred from $40 \mathrm{keV}$ electrons to carbon atoms, calculated with or without considering thermal vibration.

Figure 3(a). DCS as a function of selected $E_{k}$ and $E_{d}$ values, and (b) threshold $E_{t h}$ as a function of $E_{d}$. The solid lines in (a) are obtained using Eq. (8) and the solid lines in (b) are obtained using Eq. (9).

Figure 4 (a) DCS as a function of electron beam energy $E_{k}$, calculated by using displacement energy of 22 $\mathrm{eV}$ and at different temperatures when thermal vibration is considered, and (b) comparisons of DCS calculated with or without thermal vibration at $300 \mathrm{~K}$.

Figure 5. Displacement energies as a function of tube diameters in SWNTs, calculated by MD simulations.

Figure 6. DCS values as a function of SWNT tube diameters for temperatures of 200, 300, 400, and 500 $\mathrm{K}$, and for electron beam energies of (a) $80 \mathrm{keV}$, (b) $100 \mathrm{keV}$, (c) $120 \mathrm{keV}$, and (d) $200 \mathrm{keV}$, respectively. 